\title{
COMMUNICATION
}

\section{Diagnostic d'un kératokyste 5 ans après une germectomie de dent de sagesse}

\author{
Lenfant $\mathrm{B}^{*}$, Cloitre $\mathrm{A}^{* *}$, Lemoine $\mathrm{S}^{*}$, Lecomte $\mathrm{V}^{* *}$, Malthièry $\mathrm{E}^{* *}$, Boedec $\mathrm{A}^{* *}$, \\ Dauzat $\mathrm{A}^{* *}$, Kimakhe $\mathrm{S}^{* *}$, Lesclous $\mathrm{P}^{* *}$ \\ * DESCO, Unité Fonctionnelle de Chirurgie Orale, CHU de Nantes, 1 place Alexis Ricordeau, 44042 Nantes \\ ** Unité Fonctionnelle de Chirurgie Orale, CHU de Nantes, 1 place Alexis Ricordeau, 44042 Nantes
}

\section{Introduction}

Les kératokystes odontogéniques sont des lésions des maxillaires engendrées par la dégénérescence de la lame dentaire, à prédominance légèrement masculine (sex ratio 1,42/1) dont le diagnostic est plutôt porté dans la deuxième et troisième décade (1). Ils siègent principalement dans la région molaire mandibulaire. Parfois, une dent incluse y est associée.

Observation

Une jeune femme de 20 ans avait été adressée par son praticien traitant pour une lésion osseuse radioclaire angulo-mandibulaire droite. Cette patiente avait consulté ce praticien pour des douleurs légères à modérées (EVA $30 \mathrm{~mm}$ ) apparues trois semaines auparavant et efficacement combattues par une automédication antalgique (paracétamol) de manière sporadique. Elle rapportait aussi une sensation de dysgueusie. Elle n'avait pas d'antécédents généraux notables. Elle faisait régulièrement des otites à droites, et se plaignait de craquements au niveau de l'ATM droite depuis 3 ans. Elle indiquait que ses dents de sagesses avaient été avulsées 5 ans auparavant. La radiographie panoramique de l'époque ne mettait aucune image kystique en évidence.

La radiographie panoramique faite le jour de la consultation objectivait une volumineuse lésion limitée par un fin liseré ostéocondensant, le contenu semblait homogène ; elle s'étendait de la racine distale de la 47 jusqu'à la moitié de la hauteur de la branche montante. L'examen tridimentionnel (CBCT) réalisé le jour même, confirmait une lésion bien circonscrite, sans effacement des corticales osseuses, et avec refoulement basal du nerf alvéolaire inférieur. Un grand axe de $30 \mathrm{~mm}$ avait été mesuré.

L'exérèse chirurgicale de la lésion avait été réalisée par voie endo-buccale sous anesthésie générale. Les suites post-opératoires furent simples, si ce n'était une hypoesthésie labio-mentonnière modérée, qui a régressé totalement au bout de quelques semaines. Une radiographie panoramique de contrôle avait été réalisée à 6 mois, montrant une cicatrisation osseuse convenable.

L'analyse histologique de la pièce opératoire a permis de poser le diagnostic d'un kératokyste de type orthokératosique.

Discussion

L'intérêt de ce cas clinique est la mise en évidence de l'apparition d'un kératokyste au niveau du site d'avulsion du germe d'une dent de sagesse réalisée 5 ans auparavant, que l'imagerie initiale ne laissait nullement supposer. Une revue de la littérature n'a pas permis de trouver d'autres cas semblables d'apparition de lésion ostéolytique de type kératokystique après une telle germectomie. Il est à noter que le type histologique de l'orthokératokyste diagnostiqué dans ce cas clinique est rare $(3,5 \%$ des kératokystes (2)). Bien que bénins, ils sont localement agressifs et ont tendance à la récidive (de $17 \%$ à $56 \%$ (3)). 
$63^{\text {ème }}$ Congrès de la SFCO

Conclusion

Un suivi radiologique doit être instauré pour intercepter au plus tôt une éventuelle récidive. Références

(1) Myoung et al. (2000)

(2) El-Hajj (1996)

(3) Blanas et Freund (2000)

Nom et adresse du conférencier

Benoit LENFANT

DESCO, Unité Fonctionnelle de Chirurgie Orale, CHU de Nantes 1

Place Alexis Ricordeau

44042 Nantes (France)

lenfant.b@hotmail.fr 